\title{
INTRAVENTRICULAR SEPTAL LIPOMA: A RARE CASE OF INTRACARDIAC LIPOMA
}

Nanivadekar AA ${ }^{1}$, Maganlal $\mathrm{RN}^{1}$, Pol AA ${ }^{2}$

${ }^{1}$ Ruby Hall Clinic, Pune

${ }^{2}$ AJ Institute of Medical Science and Research Centre, Mangalore

DOI: http://doi.org/10.4038/sljr.v4i1.70

Keywords: primary cardiac tumours, Cardiac Lipoma, Lipomatous hypertrophy of the atrial septum, Cardiac Pathology, Cardiac Surgery

\section{Introduction}

Cardiac lipomas are the rarest forms of benign primary cardiac neoplasms. ${ }^{1}$ The common primary tumors of the heart are myxomas, and papillary fibroelastomas. The former is generally found in the left atrium. All these tumors consist of $5 \%$ of total primary cardiac tumors. The rest are known to be metastatic. ${ }^{2}$ These tumors have no defined age or gender-wise distribution. Of note, the most common cardiac tumor in children is rhabdomyoma. In most of the cases, the patient remains asymptomatic and even the large tumor is incidentally discovered. However, lipomas can be symptomatic depending on their location within the heart, which is mostly right atrium and the left ventricle. Almost half of them originate from the subendocardium and rest originates from either subepicardium (25\%) or myocardium $(25 \%)$. All of these vary greatly in size ${ }^{3}$. This article is on a rare case of interventricular septal lipoma where the patient presented with intermittent dizziness and recurrent episode of vomiting.

\section{Case report}

A 37 year old male presented with recurrent episodes of intermittent dizziness for several days and three episodes of vomiting. He had no history of diabetes or hypertension. Dizziness was non-exertional in nature and was independent of head and neck movement or any postural changes. Moreover, the dizziness was not related to meals.

Corresponding Author: Nanivadekar AA ${ }^{1}$ (avinash.nanivadekar@gmail.com)

https://orcid.org/0000-0001-7563-302X

This is an open-access article distributed under the terms of the Creative Commons Attribution 4.0 International License, which permits unrestricted use, distribution and reproduction in any medium provided the original author and source are credited. 
He did not have chest pain, dyspnoea, orthopnoea, paroxysmal nocturnal dyspnoea, leg swelling, palpitations or hearing loss or any significant family history. The patient denied smoking, alcohol or substance abuse.

On presentation the vital signs were normal with heart rate 80 beats/min in regular rhythm, blood pressure170/110 $\mathrm{mm} \mathrm{Hg}$, respiratory rate $18 / \mathrm{min}$ and spo2: $99 \%$ on room air. No orthostatic changes were observed in his blood pressure and heart rates. Physical examination revealed normal cardiovascular, respiratory and nervous system findings. Laboratory tests revealed a normal complete blood count, kidney function, liver function tests and electrolyte composition.

Differential diagnosis at this stage included cardiac lipoma. Lipomatous hypertrophy of interatrial/ventricular septum, atrial myxoma and papillary fibroelastoma.

The patient was subjected to a standard 12 lead electrocardiogram (ECG) which showed normal sinus rhythm with partial left bundle branch block. As expected, a transthoracic echocardiogram showed normal left ventricular systolic function with a hyperechoic shadow in the left ventricular surface on interventricular septum (Fig.1 and 2). No Left ventricular outflow tract obstruction was observed. Mild left ventricular hypertrophy with LVEF - $60 \%$ was found. Based on the findings of the primary assessment, a cardiac magnetic resonance imaging $(\mathrm{CMR})$ with cardiac computerized tomography (CCT) was performed.

For cardiac MRI, a 3 Tesla Phillips MRI with multiplanar and multiecho sequences was employed. Cardiac CT was performed for correlation. A hypodense lesion (-65 HU) was detected at the medial aspect of the intraventricular septum in the left ventricle which extended superiorly into the medial chordae tendinae involving the papillary muscle. The dimension of the lesion was 4.5 x $1.5 \mathrm{~cm}$ (Fig. 5 and Fig. 6). The lesion did not result in any constriction or obstruction to the outflow tract of the left ventricle. The lesion had abundant fatty contents on MRI. No abnormal early or late enhancement was found when the patient was subjected to Gadolinium contrast examination (Fig. 4). Radiological investigation revealed no sign of calcification or hemorrhage. No abnormal thickening or bowing was found in interventricular septum. The cardiac chambers were otherwise normal. CT coronary angiography showed the 
anomalous origin of left circumplex artery from right coronary cusp interposed between the aorta and pulmonary artery (Fig. 7). Left anterior descending artery, right coronary artery, pulmonary artery and aorta appeared normal in course and caliber. There was no evidence of pericardial or pleural effusion and the pericardial fat pad was preserved.

The patient was treated conservatively instead of surgical resection and planned for follow up after 6 months. The patient remains asymptomatic up to date.

\section{Discussion}

Cardiac lipomas are benign tumors of the heart that can occur in any age group with a strong prevalence in the age group of 40-60 years. ${ }^{3}$ Usually, this condition is asymptomatic and thus remains undetected unless discovered incidentally. Patients can present with a variety of symptoms depending on the location of the tumor within the heart. Tumors in subepicardial region can cause angina as the huge mass of the tumor compresses the coronary arteries. Moreover, they can reduce systolic function by compressing on the left ventricle. Similarly, tumors in the myocardium can infiltrate the cardiac electrical circuit resulting in arrhythmogenesis ${ }^{4}$. The size of the tumor and the chamber involved will determine whether it will lead to an obstruction of flow followed by congestive heart failure. Formation of an embolus is a very rare event. A simple and non-invasive diagnostic approach to detect lipoma is transthoracic echocardiographic (TTE) examination. However, TTE is neither able to visualize smaller tumors nor can it conclusively differentiate between lipomas and other primary tumors of the heart. ${ }^{5}$ Keeping in mind this limitation of TTE, CCT and CMR imaging can be used to gather further information. Differentiation between different classes of cardiac tumors is an important goal of diagnostic imaging. Lipomas appear to have homogenous high signal intensity with no enhancement on T1 and T2 weighted images whereas liposarcomas typically contain solid, enhancing components intermixed with areas of the fat signal. Similarly, lipomatous hypertrophy of the interatrial septum is another differential diagnosis of cardiac fatty masses. However, lipomatous hypertrophy includes hyperplasia of adipocytes as opposed to hypertrophy as the name suggests. These are unencapsulated, sparing the fossa ovalis and thus bear a dumbbell shape ${ }^{5}$. 
In our case, the patient presented with a history of recent recurrent intermittent dizziness and vomiting with no other complications. Echocardiography followed by $\mathrm{CMR}$ and $\mathrm{CCT}$ confirmed the diagnosis of lipomas as it showed characteristic radiological features. In general, surgical resection is the mainstay for treating cardiac lipoma. However, the patient needed no further treatment and was treated conservatively.

\section{Conclusion}

This case report emphasizes the utility of the diagnostic approach using transthoracic echocardiogram in combination with CCT and cardiac MRI to accurately characterize cardiac lipomas. This diagnostic approach provides an accurate explanation of the imaging findings and the etiological information of the disease which in turn helps in deciding the management strategy.

\section{References}

1. Heath D. Pathology of cardiac tumors. Am J Cardiol. 1968 Mar 1;21(3):31527.

2. Silverman NA. Primary cardiac tumors. Ann Surg. 1980 Feb;191(2):127-38.

3. Ismail I, Al-Khafaji K, Mutyala M, Aggarwal S, Cotter W, Hakim H, et al. Cardiac lipoma. J Community Hosp Intern Med Perspect. 2015;5(5):28449.

4. D'Souza J, Shah R, Abbass A, Burt JR, Goud A, Dahagam C. Invasive Cardiac Lipoma: a case report and review of literature. BMC Cardiovasc Disord [Internet]. 2017 Dec [cited 2018 Feb 25];17(1). Available from:

http://bmccardiovascdisord.biomedcentr al.com/articles/10.1186/s12872-0160465-2

5. Barbuto L, Ponsiglione A, Del Vecchio W, Altiero M, Rossi G, De Rosa D, et al. Humongous right atrial lipoma: a correlative CT and MR case report. Quant Imaging Med Surg. 2015;5(5):774. 


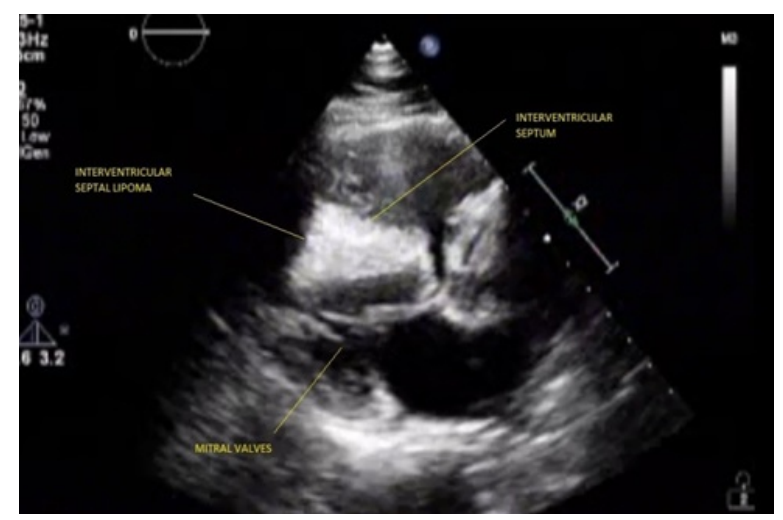

Figure 1: Transthoracic echocardiogram (TTE) imaging of the lipoma: the parasternal long axis view of shows hyperechoic mass originating from the interventricular septum in left ventricle

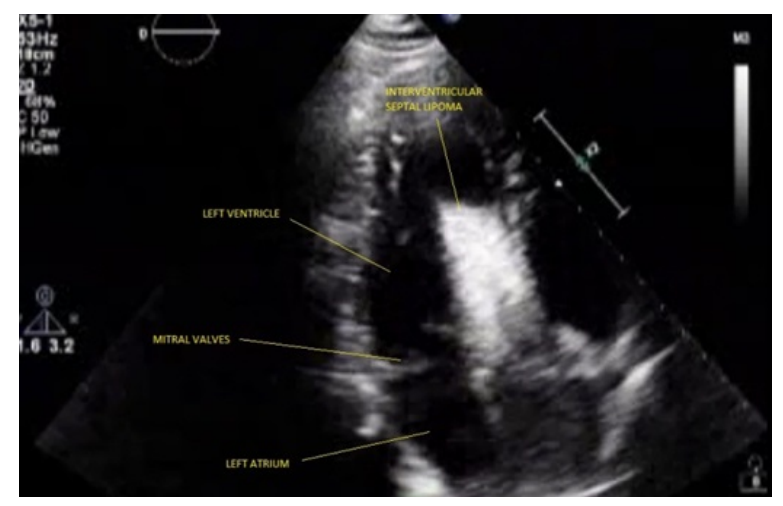

Figure 2: The four chamber view of interventricular septum in the transthoracic echocardiogram (TTE) imaging shows a hyperechoic mass attached to interventricular septum in left ventricle.

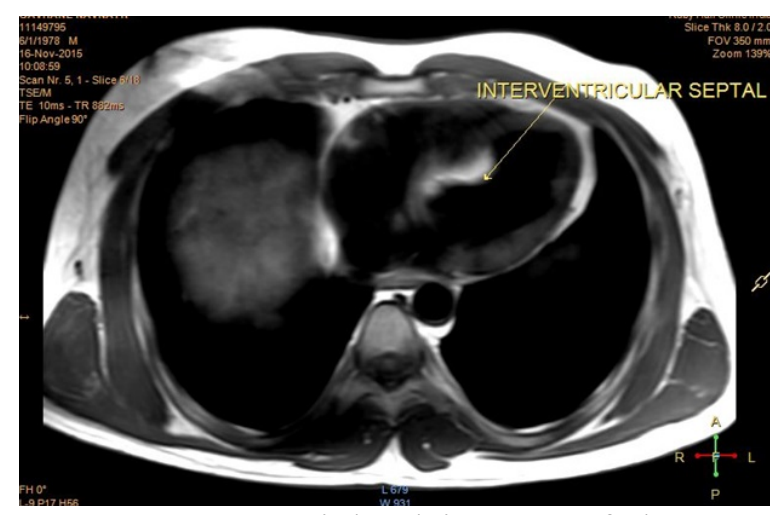

Figure 3: T1 weighted images of the ventricle (Axilla View) in cardiac magnetic resonance imaging (CMR) shows hyperintense lesion attached to the interventricular septum. 


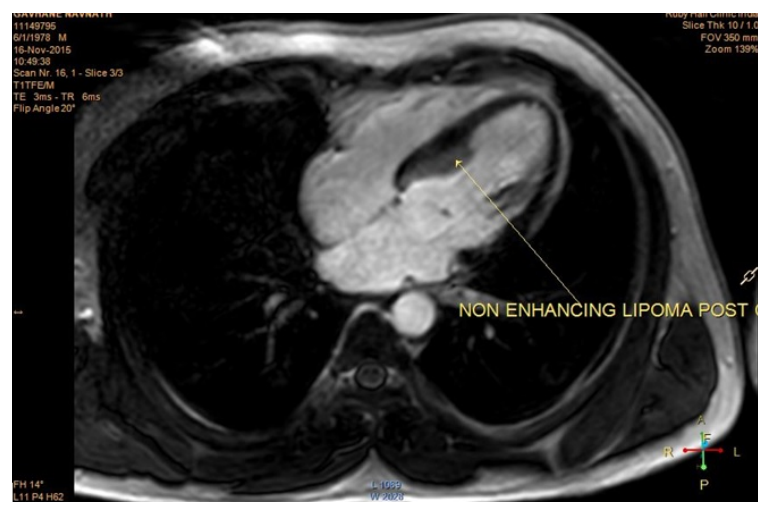

Figure 4: Cardiac magnetic resonance imaging studies show nonenhancement of the lesion on post gadolinium contrast study (axial view)

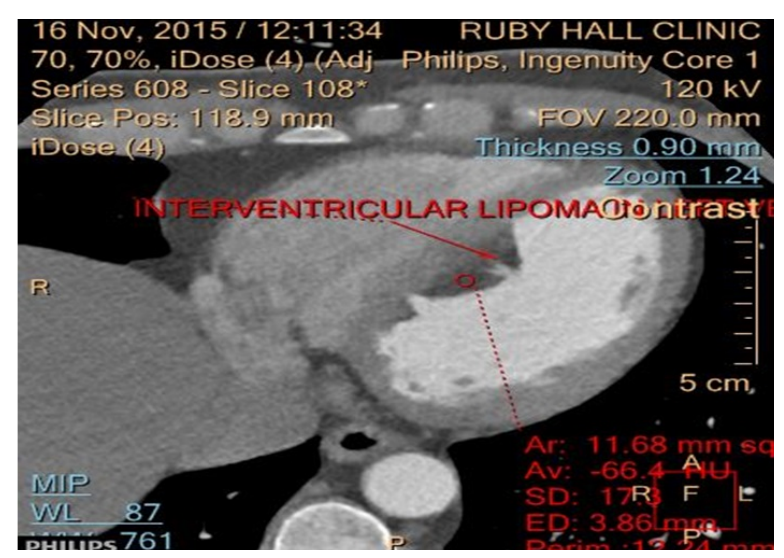

Figure 5: The axial view of interventricular septum of left ventricle in cardiac computerized tomography (CCT) studies shows presence of hypodense lesion (HU value of -65) along medial aspect of interventricular septum.

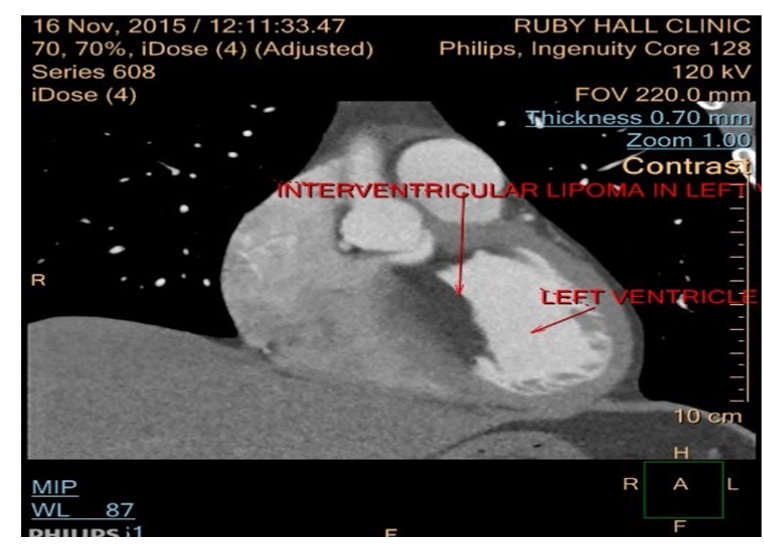

Figure 6: Cardiac computerized tomography (CCT) in coronal view showing the invasion of the hypodense lesion in to the medial chordae tendinae and involving the papillary muscle 


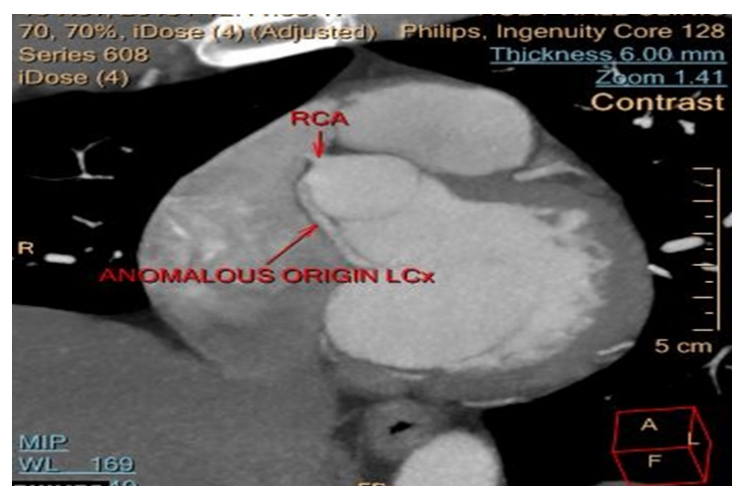

Figure 7 : Cardiac computerized tomography (CCT) imaging showing the anomalous origin of LCx from right coronary cusp interposed between the aorta and pulmonary artery. 